\title{
Freeze Fracture Electron Microscopy of Dilute Lamellar and Anomalous Isotropic $\left(\mathrm{L}_{3}\right)$ Phases
}

\author{
R. Strey ${ }^{*}, \dagger$ W. Jahn, ${ }^{\dagger}$ G. Porte, ${ }^{\ddagger}, \S$ and P. Bassereau ${ }^{\ddagger}$ \\ Max Planck Institut für Biophysikalische Chemie, Postfach 2841, 3400 Göttingen, FRG, \\ U.S.T.L. Groupe de Dynamique des Phases Condensees, Place E.-Bataillion, 34060 \\ Montpellier, France, and Institute for Theoretical Physics, University of California, \\ Santa Barbara, California 93106
}

Received January 31, 1990. In Final Form: April 27, 1990

\begin{abstract}
We show images of dilute lamellar $\left(\mathrm{L}_{\alpha}\right)$ and anomalous isotropic $\left(\mathrm{L}_{3}\right)$ phases obtained by freeze fracture electron microscopy. The images of the $L_{\alpha}$ phase show stacks of bilayers. In some parts, undulations of the bilayers may be seen. The images from the $L_{3}$ phase show an apparently bicontinuous network of two aqueous subvolumes separated by a random bilayer network. Saddle point structures with a mean curvature close to zero and a negative Gaussian curvature are observed. The bilayers are separated by several hundred angstroms, and the repeat distances seen on the images agree with those measured previously by SANS. Furthermore, the transition from $\mathrm{L}_{\alpha}$ to $\mathrm{L}_{3}$ through the intermediate state of passage formation seems to be captured.
\end{abstract}

\section{Introduction}

Images of the microstructure of microemulsions by freeze fracture electron microscopy $(\text { FFEM })^{1}$ revealed a close to zero mean curvature structure of the surfactant monolayers between the water and oil domains. Systematic changes in the local curvature of the microstructure by varying the water-to-oil ratio could be demonstrated. Apparently, the changes in microstructure due to cooling process had been minimized by using relatively large scale structures (of the order of $500 \AA$ ). The observation of a zero mean curvature and negative Gaussian curvature of the surfactant monolayers can be considered a strong indication for bicontinuity ${ }^{2}$ of the microemulsions. Structural predictions from phenomenological theory compared favorably with the images. ${ }^{3}$ Recently, the same theory has been applied to the anomalous isotropic $\mathrm{L}_{3}$ phase. ${ }^{4}$ It describes this phase as a random surfactant bilayer network separating two equivalent water-continuous subvolumes. Apparently, the structure of such systems may be described as an ensemble of nonintersecting films ${ }^{5}$ where structural transformations are driven by the interfacial and bending properties of the mono- and bilayers. ${ }^{6}$ As model systems for such ensembles, the structure of the dilute lamellar and the $\mathrm{L}_{3}$ phase is currently being investigated. The $\mathrm{L}_{3}$ phase is always observed in the vicinity of a dilute lamellar phase, as can be seen most clearly in binary water-nonionic surfactant systems. ${ }^{7}$ Scattering techniques have recently provided precise data on the length scales of structure of the dilute lamellar phase for a number of systems.., $9 \mathrm{We}$, therefore, saw the chance to apply the FFEM method to

* To whom correspondence should be addressed.

† Max Planck Institut für Biophysikalische Chemie.

¿ U.S.T.L. Groupe de Dynamique des Phases Condensees.

8 Institute for Theoretical Physics.

(1) Jahn, W.; Strey, R. J. Phys. Chem. 1988, 92, 2294.

(2) Lindman, B.; Shinoda, K.; Olsson, U.; Anderson, D.; Karlstöm, G.; Wennerström, H. Colloids Surf. 1989, 38, 205.

(3) Milner, S. T.; Safran, S. A.; Cates, M. E.; Andelman, D.; Roux, D. J. Phys. (Les Ulis, Fr.) 1988, 49, 1065.

(4) Cates, M. E.; Roux, D.; Andelman, D.; Milner, S. T.; Safran, s. A. Europhys. Lett. 1988, 5, 733 .

(5) Huse, D. A.; Leibler, S. J. Phys. (Les Ulis, Fr.) 1988, 49, 605.

(6) Porte, G.; A ppell, J.; Bassereau, P.; Marignan, J.J. Phys. (Les Ulis, Fr.) $1989,50,1335$.

(7) Strey, R.; Schomäcker, R.; Roux, D.; Nallet, F.; Olsson, U. J. Chem. Soc., Faraday Trans. 1990, 86, 2253. water-swollen $\mathrm{L}_{\alpha}$ and $\mathrm{L}_{3}$ phases where the characteristic length scales are known. Cryofixation of samples identical in composition with those studied previously by smallangle neutron scattering (SANS) $)^{8,10}$ permits a quantitative comparison of the spacings. Local structures from smallangle scattering ${ }^{11}$ are obtained as statistical averages, while microscopy may yield additional information on the details of the local structure.

Here, we present images of the local structure by FFEM in the dilute lamellar and $\mathrm{L}_{3}$ phases for the binary system water-didodecylammonium bromide and the quaternary system water $(\mathrm{NaCl})-1$-hexanol-cetylpyridinium chloride. Specifically, the latter system has been selected because analogies in the phase behavior suggest that there are also general patterns of the microstructure common to the more complex multicomponent mixtures ${ }^{6,12}$ and the simpler binary water-nonionic surfactant systems. ${ }^{7}$ The temperature sensitivity of the latter, however, poses a problem to cryofixation. Actually, before obtaining the results presented below we investigated the dilute lamellar and $\mathrm{L}_{3}$ phases of some binary systems, water-nonionic amphiphile, including ${ }^{7}$ water- $\mathrm{C}_{12} \mathrm{E}_{5}$. However, due to the strong temperature dependence and the elevated temperatures (around $55^{\circ} \mathrm{C}$ for water $-\mathrm{C}_{12} \mathrm{E}_{5}$ ), no reliable results were obtained. Also, for the dilute lamellar phases in the quaternary systems water-1-pentanol-SDS- $\mathrm{NaCl}$ and water-dodecane-pentanol-SDS previously studied by Bordeaux, ${ }^{12}$ cryofixation was not successful.

\section{Experimental Section}

Materials. The didodecylammonium bromide (DDABr) was the same as that used in ref 1 . The samples for the cetylpyridinium chloride (CPyCl) system were from the same components as used previously for the SANS study. ${ }^{8}$ The water was doubly distilled.

Phase Behavior. It has previously been shown ${ }^{6}$ that the cosurfactant/surfactant molar ratio $(\delta)$ plays a role similar to that of temperature $(T)$ in binary nonionic surfactant systems. ${ }^{7}$ In-

(8) Porte, G.; Marignan, J.; Bassereau, P.; May, R. Europhys. Lett. 1988, $7,713$.

(9) Roux, D.; Safinaya, C. R. J. Phys. (Les Ulis, Fr.) 1988, 49, 307.

(10) Porte, G.; Mariman, J.; Bassereau, P.; May, R. J. Phys. (Les Ulis, Fr.) $1988,49,511$

(11) Marignan, J.; Appell, J.; Bassereau, P.; Porte, G.; May, R. P. J. Phys. (Les Ulis, Fr.) 1989, $50,3553$.

(12) Gazeau, D.; Bellocq, A. M.; Zemb, T.; Roux, D. Europhys. Lett. $1989,9,447$. 


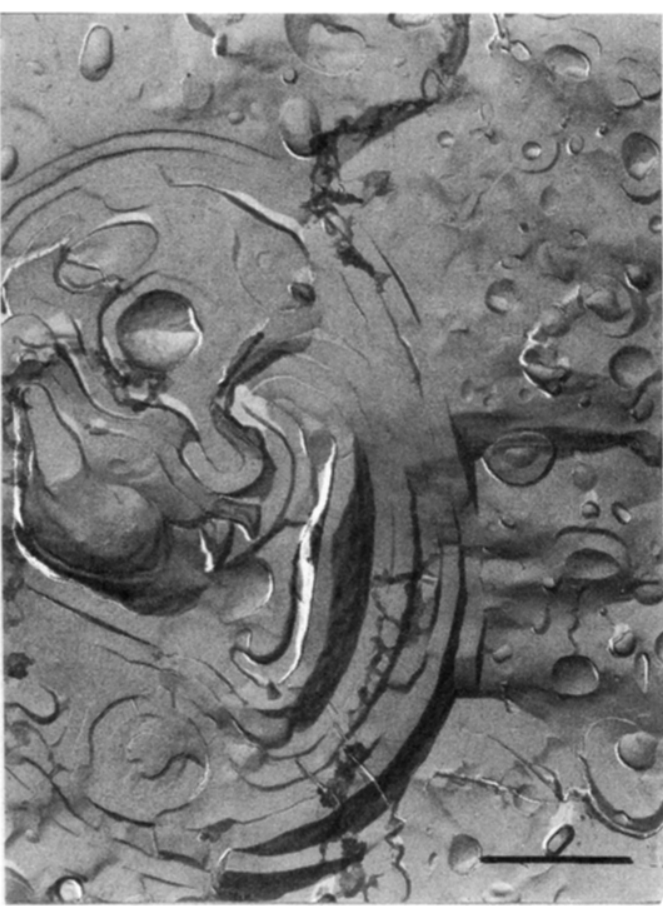

Figure 1. Dilute lamellar phase in the binary water-DDABr system: $\phi_{\mathrm{w}}=0.98, \mathrm{bar} \cong 1 \mu \mathrm{m}$. Note the large spacing of the bilayers of $d=1250 \AA$.

creasing the ratio changes the surfactant combination from hydrophilic to lipophilic, and the phase sequence $\mathrm{L}_{1}-\mathrm{L}_{\alpha}-\mathrm{L}_{3}$ is observed (as with $T$ in nonionic systems). This phase sequence is accompanied by a sphere-to-rod-to-bilayer transition. This trend was demonstrated by SANS $^{9}$ and may be interpreted as a change of the spontaneous curvature $c_{0}$ of the monolayers toward brine (an argument that shall be used later on). Accordingly, the samples can be characterized in pseudobinary approximation by their brine volume fraction $\phi_{\mathrm{w}}$ and the 1 -hexanol/CPyCl ratio $\delta$. The anisotropy and streaming birefringence were checked with crossed polarizers prior to freezing the sample.

FFEM. Cryofixation involves a number of subsequent preparation steps: the solutions are rapidly frozen, the sample is fractured, the fracture face is replicated, and the replica is studied by transmission electron microscopy. The first step is crucial: starting from around ambient temperature, roughly $100 \mathrm{~K}$ have to be passed as fast as possible; that is, the cooling rate $-\mathrm{d} T / \mathrm{d} t$ has to be as high as possible. This minimizes structural changes (e.g., the local curvature of the bilayers). Furthermore, during the freezing process the formation of ice crystals should be avoided. Otherwise, the spatial arrangement of the dispersed bilayers will be distorted. The sandwich technique, as we have it adapted to studying fluid microstructures, has been described previously. ${ }^{1}$ It has been modified in that we subjected a drop of the solution directly from a pipet to one of the supporting copper disks at room temperature $\left(20^{\circ} \mathrm{C}\right)$. We took care that there was as little time as possible for evaporation. Within about $5 \mathrm{~s}$ the EM grid and second support disk were added, and thereby the sample was squashed into the meshs of the grid. The sandwich was grabbed by tweezers attached to a plunging device and frozen. The cooling rate is about $10^{4} \mathrm{~K} / \mathrm{s}$. From application of the drop to freezing we allowed about $30 \mathrm{~s}$ for reequilibration after shear during assembly of the sandwich.

Electron microscopy of the replicas requires discrimination between artifacts and what might come close to reality. Vast portions of the replica are often spoiled by ruptures of the carbon film and artifacts, such as surface fractures, phase separation, slow cooling, etc. If, however, we find structures which occur reproducibly on separate grids and which are expected on theoretical grounds and/or other experimental evidence, we consider them as probably close to reality.

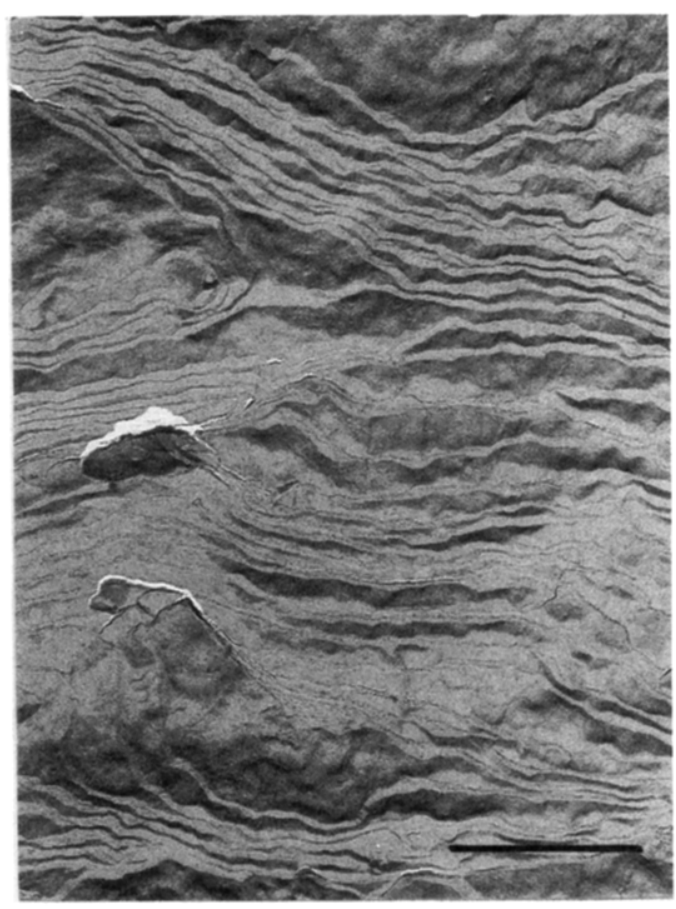

Figure 2. Dilute lamellar phase in the quaternary water $(0.2 \mathrm{~m}$ $\mathrm{NaCl}$ )-1-hexanol-CPyCl system: $\phi_{\mathrm{w}}=0.933, d=360 \AA$ (from $\mathrm{SANS})$, bar $\cong 1 \mu \mathrm{m}$. Note the oblique fracture disclosing the undulating nature of the stacked bilayers.

\section{Results}

As mentioned in the Introduction, several systems had to be tested before the first successful results were obtained. As an example, we show in Figure 1 the image for a solution of $\phi_{\mathrm{w}}=0.98$ for the binary system water-DDABr. The lamellar structure is found to be disrupted in multilamellar vesicles probably as a result of the shear stress during preparation. Nevertheless, a well-defined spacing of the bilayers of $\bar{d}=1250 \AA$ is observed presumably caused by long-range electrostatic repulsion. Furthermore, the freezing process did not destroy the bilayers. Our results support the findings of Talmon ${ }^{13}$ on the same system using direct imaging (DI). There the samples were investigated in the frozen hydrated state; well-separated bilayers often organized in multilamellar vesicles have also been shown.

An example for a system stabilized by steric repulsion is the system water $(0.2 \mathrm{~m} \mathrm{NaCl})-1$-hexanol-CPyCl. The images shown in Figures 2 and 3 were obtained from samples with intermembrane distances of $360 \AA\left(\phi_{\mathrm{w}}=\right.$ $0.933, \delta=3.43)$ and $740 \AA\left(\phi_{\mathrm{w}}=0.962, \delta=3.43\right)$ as measured by SANS. ${ }^{8}$ Clearly the multilayered structure is seen. The fracture on Figure 2 has occurred under an oblique angle. Where the fracture occurred along the bilayer midplane, undulations of the bilayers seem to be captured. Figure 3 permits the determination of the average spacing over nine water layers (between arrows in Figure 3$) ; \bar{d}=740( \pm 40) \AA$ in agreement with the lamellar spacings from SANS.

Figure 4 shows the structure encountered upon freezing a sample $\left(\phi_{\mathrm{w}}=0.933, \delta=3.64\right)$ in the $\mathrm{L}_{3}$ phase. The fracture through the water domains is planar. The structural distance is consistent with SANS and corresponds roughly to the diameter of the passages in the $3 \mathrm{D}$ network of the bilayers. Where the fracture occurred along the bilayer, saddle point local shapes can be found. The typical shape of the bilayer one frequently observes is drawn as an artist's impression in Figure 5. It resembles

(13) Talmon, Y. Colloids Surf. 1986, 19, 237. 


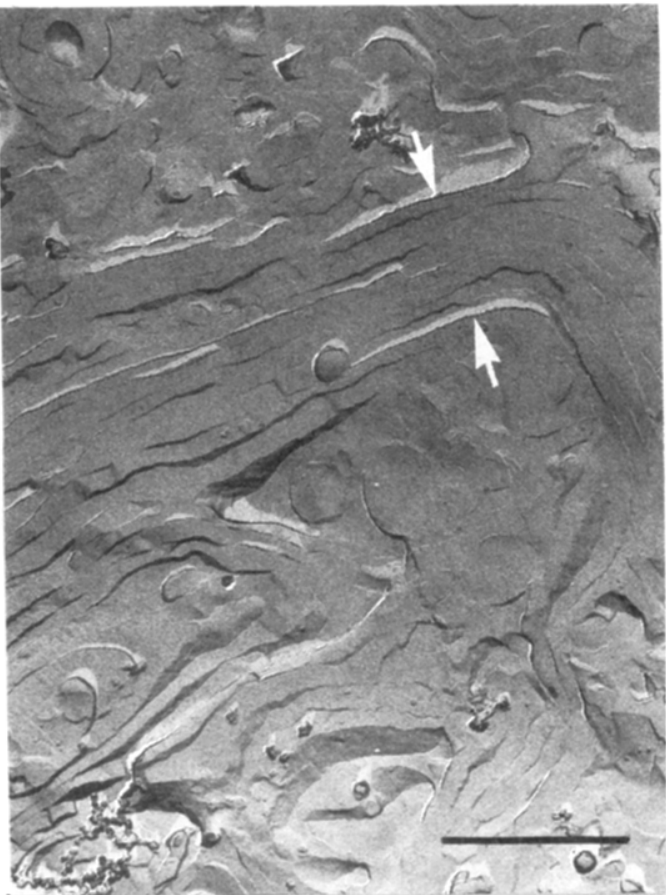

Figure 3. Dilute lamellar phase in the quaternary water $(0.2 \mathrm{~m}$ $\mathrm{NaCl}$ )-1-hexanol-CPyCl system: $\phi_{\mathrm{w}}=0.962, d=740 \AA$ (from SANS), bar $\cong 1 \mu \mathrm{m}$. Note the fracture normal to the bilayers permits one to measure the bilayer spacing $\bar{d}=740( \pm 40) \AA$ between arrows.

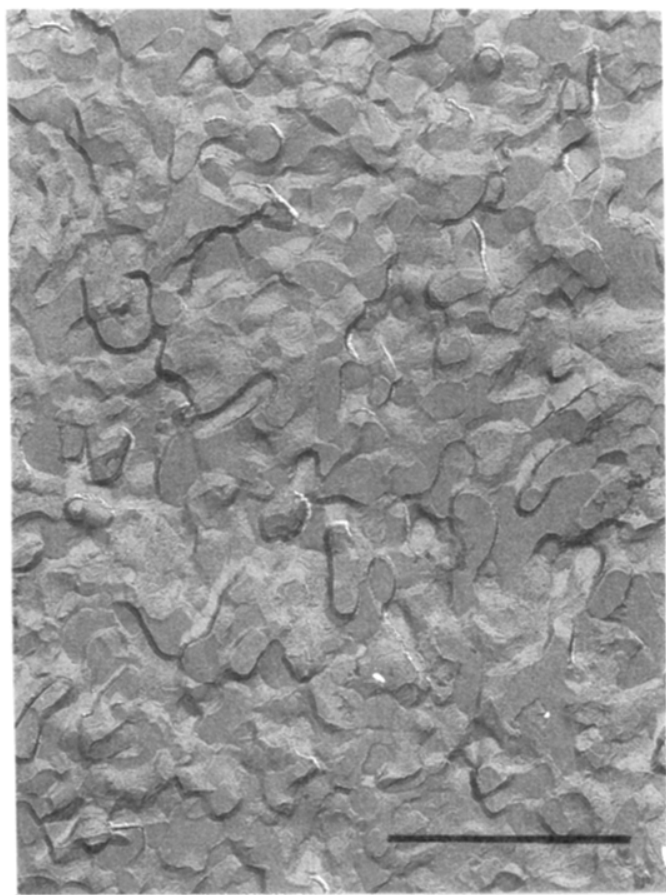

Figure 4. Anomalous $\mathrm{L}_{3}$ phase in the quaternary water $(0.2 \mathrm{~m}$ $\mathrm{NaCl}$ )-1-hexanol-CPyCl system: $\phi_{\mathrm{w}}=0.933$, bar $\cong 1 \mu \mathrm{m}$. Note the fracture through the random bilayer network either occurs planar through bulk water or follows the bilayer midplane.

a randomly connected "plumbers nightmare"..$^{5}$ Note that we do not want to imply a phonon-like deformation of a cubic mesophase but rather a more randomly connected bilayer network in order to be consistent with the scattering properties of the $\mathrm{L}_{3}$ phases.

In order to support this vision, we show in Figure 6 a stereopair of images of the $\mathrm{L}_{3}$ structure. The reader is invited to use stereoglasses to convince himself of the threedimensionality of the structure. In some portions of Figure 6 (arrow), the saddle-shaped bilayer surface between the

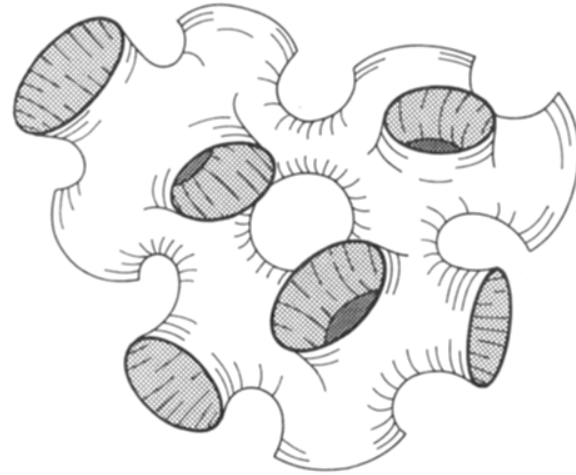

Figure 5. Schematic drawing of the randomly connected bilayer network proposed for the $\mathrm{L}_{3}$ phase.
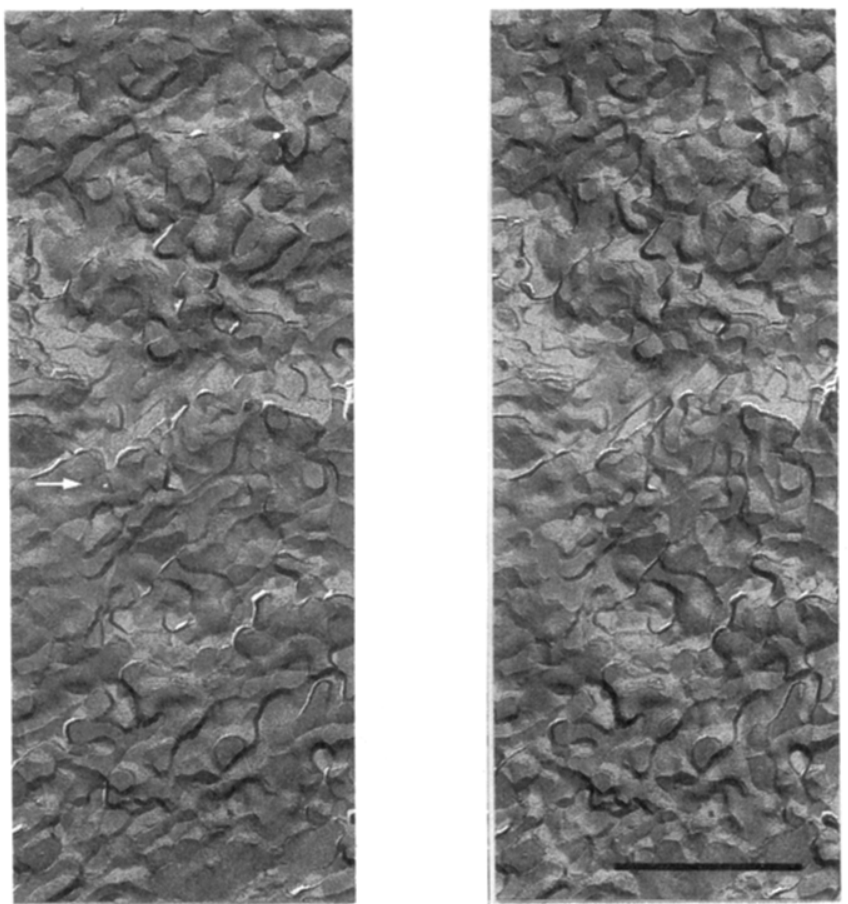

Figure 6. Stereopair of sample in the $\mathrm{L}_{3}$ phase. Use stereoglasses to obtain 3D impression. Note that a bilayer with zero mean curvature and negative Gaussian curvature separates two equivalent water subvolumes (arrow).

two intertwined brine subvolumes is disclosed.

Figure 7 is specially interesting since it exhibits a structure with features specific both to $\mathrm{L}_{\alpha}$ and $\mathrm{L}_{3}$. It is obtained from the sample $\left(\phi_{\mathrm{w}}=0.933, \delta=3.43\right)$ with a composition close to the $\mathrm{L}_{\alpha}$ to $\mathrm{L}_{3}$ boundary. The image is taken from a different region on the same grid from which Figure 2 was taken. The observed structure still retains the multilayered character of $\mathrm{L}_{\alpha}$, but the inplane smoothness of the bilayers is now disrupted by a large amount of passages normal to the lamellar stacking.

Figure 8 shows in a schematic drawing a stack of bilayers with passages, as has recently been discussed. ${ }^{5}$ This intermediate structure is probably an unstable state between the stable $\mathrm{L}_{\alpha}$ and $\mathrm{L}_{3}$ phases: SANS patterns obtained with a well-oriented $\mathrm{L}_{\alpha}$ sample of identical composition at $20^{\circ} \mathrm{C}$ did not reveal a significant density of passages. Thus, the intermediate structure either arises from local compositional variations or is transiently induced during the cooling process and then frozen. Its occurrence, however, suggests interesting insights in the $\mathrm{L}_{\alpha}$ to $\mathrm{L}_{3}$ structural transformation.

To elucidate this point further, we show in Figure 9 that a locally slightly different composition or thermal history 


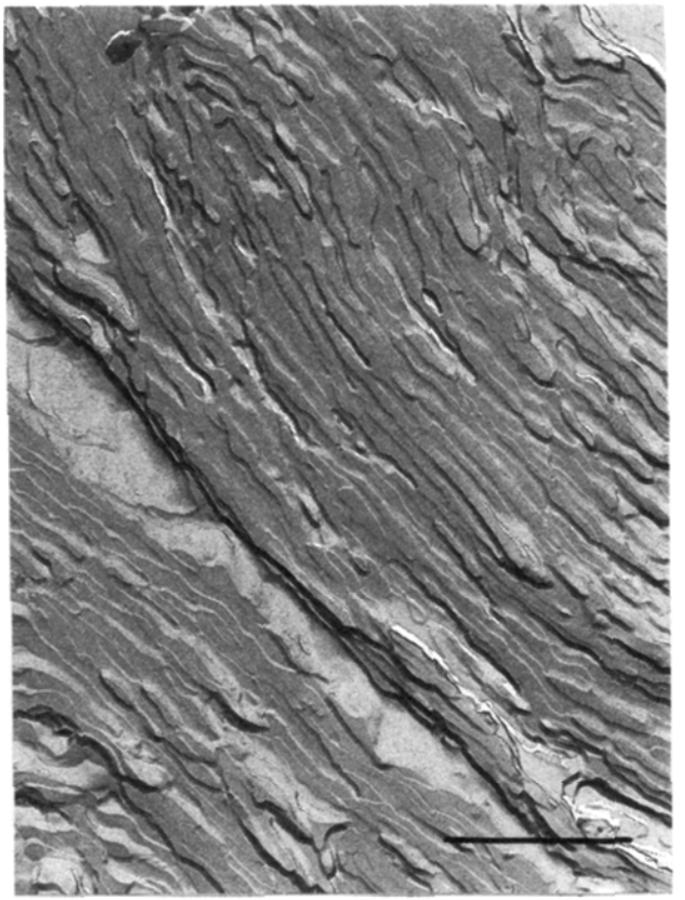

Figure 7. Dilute lamellar phase becoming perforated by passages.

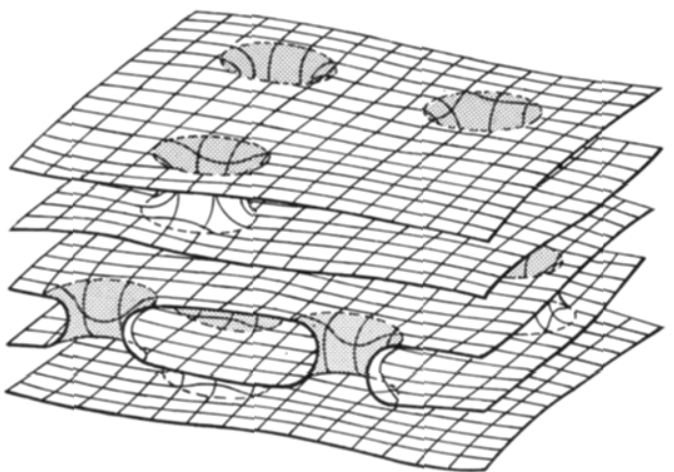

Figure 8. Schematic drawing of transient state of passage formation. $^{5}$

permits one to see the transformation from the perforated lamellar state into what resembles closely the $\mathrm{L}_{3}$ structure.

\section{Discussion}

Under favorable conditions, cryofixation of the local structure of dilute lamellar and $\mathrm{L}_{3}$ phases is possible. However, the images have to be considered with some caution, mainly because the conditions under which the preparation seemed to be successful are not clear. One might speculate that a prerequisite is the reduced temperature dependence of the local curvature of the monolayers due to mixing ionic and nonionic amphiphiles. Such mixtures are known ${ }^{14}$ to become temperature insensitive for certain mixing ratios.

On the other hand, we are able to find structures that have been postulated previously and independently on theoretical ${ }^{4,6,15}$ grounds and concluded from other experimental evidence. ${ }^{8-10,12}$ This fact supports the images.

Two basic differences between the $\mathrm{L}_{\alpha}$ and $\mathrm{L}_{3}$ structures must be considered. The first one is the range of positional order. $\mathrm{L}_{\alpha}$ shows long-range smectic order (focal conics

(14) Kahlweit, M.; Strey, R. J. Phys. Chem. 1988, 92, 1557.

(15) Anderson, D.; Wennerström, H.; Olsson, U. J. Phys. Chem. 1989, $93,4243$.

(16) Helfrich, W. Z. Naturforsch. 1978, 33a, 305.
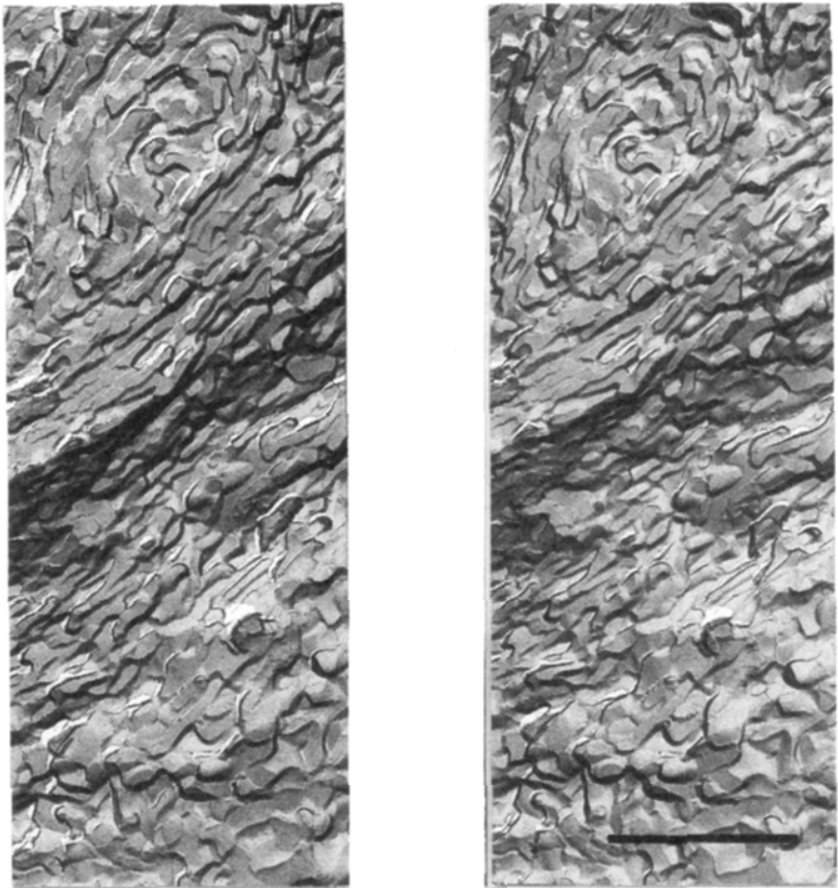

Figure 9. Stereopair of sample undergoing $\mathrm{L}_{\alpha}$ to $\mathrm{L}_{3}$ transformation (top to bottom). Use stereoglasses to obtain 3D impression. Note the structure changes from lamellar with passages (upper portion) to random bilayers with negative Gaussian curvature (lower portion).

textures), whereas $\mathrm{L}_{3}$ is a liquid-like disordered phase as indicated by the images and the scattering profiles., 90

The second one is the change in topology of the bilayer surface. In $\mathrm{L}_{\alpha}$, individual bilayers are submitted to thermal undulation fluctuations but keep the topological type of flat planes, as long as the density of passages is negligible. On the other hand, the multiconnected isotropic structure of $\mathrm{L}_{3}$ implies a very large density of passages.

One way to rationalize the $\mathrm{L}_{\alpha}$ to $\mathrm{L}_{3}$ transformation is the following argument in terms of the bending free energy of the bilayers. The simplest topological invariant associated with a given shape for a surface is the integral of the Gaussian curvature over the surface:

$$
\int_{A} c_{1} c_{2} \mathrm{~d} A=4 \pi n
$$

where $n$ is the Euler characteristic of the surface, i.e., the number of passages $\left(c_{1}\right.$ and $c_{2}$ are the two principal curvatures of the surface).

Expressing the bending elasticity contribution of the bilayers to the free energy density according to Helfrich ${ }^{16}$

$$
\mathrm{d} E_{\mathrm{el}}=\left[(1 / 2) \kappa\left(c_{1}+c_{2}\right)^{2}+\bar{\kappa} c_{1} c_{2}\right] \mathrm{d} A
$$

where $\kappa$ and $\bar{\kappa}$ are the splay and saddle splay rigidity coefficients of the bilayer, one might expect the Gaussian contribution $\left(\kappa c_{1} c_{2}\right)$ to be especially important in structural transformations involving strong topological changes. For the $\mathrm{L}_{\alpha}$ to $\mathrm{L}_{3}$ transformation, the Gaussian contribution to the difference in free energy densities is equal to $-4 \pi n_{\mathrm{L}_{3} \kappa}$, where $n_{\mathrm{L}_{3}}$ is the density of passages in the $\mathrm{L}_{3}$ phase per unit volume ( $n_{\mathrm{L}_{\alpha}}$ is essentially 0 ). So any mechanism increasing the value of $\bar{\kappa}$ should thus favor $\mathrm{L}_{3}$ compared to $\mathrm{L}_{\alpha}$. It has been shown elsewhere ${ }^{6}$ that $\bar{\kappa}$ for a bilayer varies upon changes of the spontaneous curvature $c_{0}$ of the monolayers: changes of $c_{0}$ toward brine results in increasing $\bar{\kappa}$ of the bilayer. This mechanism has been suggested ${ }^{6}$ to be the basis of the $\mathrm{L}_{\alpha}$ to $\mathrm{L}_{3}$ transformation 
in the present system upon increasing the alcohol content at constant temperature.

In Figure 7, the transformation seems to be triggered by the cooling process for samples closest to the phase boundary. This could be rationalized according to the above argument assuming that a slight change in $c_{0}$ has been induced by decreasing the temperature. Another possibility is a somewhat inhomogeneous distribution of the components, either as a result of the cooling process or the preceding preparation steps. In any case, the observation of the transient intermediate structure ${ }^{5}$ (Figures 7-9) is noteworthy. It suggests that the change in topology, that is, the proliferation of passages, takes place first. It may therefore be the driving phenomenon consistent with our interpretation based on $k$ changes. The melting of the smectic order occurs afterwards as a probable consequence of the density of passages acting as defects disrupting the former ordered state.

We note here that an alternative description of the $\mathrm{L}_{\alpha}$ to $L_{3}$ transition focuses on the role of entropy in destabilizing the lamellar structure. Whatever mechanism applies, further experiments are required with alcoholfree, e.g., the water-AOT- $\mathrm{NaCl}$ and salt-free waternonionic, surfactant systems. Such work is in progress.

The transformation of $\mathrm{L}_{\alpha}$ to a cubic mesophase in the water-DOPE system also by temperature variation has been reported. ${ }^{17}$ Illustrative models of cubic structures which are topologically similar to our Figure 5 have recently been shown. ${ }^{18}$ The sensitivity of fluid surfactant structures

(17) Shyamsunder, E.; Gruner, S. M.; Tate, M. W.; Turner, D. C.; So, P. T. Biochemistry 1988, 27, 2332.

(18) Larsson, K. J. Phys, Chem. 1989, 93, 7304. to large temperature variations is usually considered as a serious limitation of the FFEM technique. In the present case, however, it gives us the opportunity of interesting, qualitative insights on the dynamics of the $\mathrm{L}_{\alpha}$ to $\mathrm{L}_{3}$ structural transformation. However, we emphasize here again that in considering the images of expected structures one always should have in mind the finite cooling rate and its possible influence.

\section{Conclusions}

The images shown above demonstrate that it is possible to cryofix thin bilayers in the dilute lamellar and the $\mathrm{L}_{3}$ phase separated by hundreds of angstroms. Undulations in the lamellar phase and the 3D multiply connected bilayers in the $\mathrm{L}_{3}$ phase are observed. Both properties have been proposed theoretically $y^{3,5,6,9,10,15,16}$ and are currently discussed as examples of fluctuating surfaces. ${ }^{5,19}$ The fact that the images show what had been concluded from independent theoretical considerations and experimental evidence $^{8-10,12}$ is considered as support for the images.

Acknowledgment. This work was performed in the department of Prof. M. Kahlweit. W.J. and R.S. are indebted to him for his continuing support. G.P. acknowledges partial support by the National Science Foundation under Grant No. PHY82-17853, supplemented by funds from the National Aeronautics and Space Administration.

Registry No. DDABr, 24447-63-0; NaCl, 7647-14-5; CPyCl, 123-03-5; hexyl alcohol, 111-27-3.

(19) Roux, D. In Random Fluctuation and Pattern Growth: Experiments and Models; Stanley, H. E., Ostrowsky, N., Eds.; Kluwer Academic: The Netherlands, 1988; p 246. 